ORIGINAL ARTICLE

\title{
Bacteraemia prediction in emergency medical admissions: role of $C$ reactive protein
}

\section{H Wyllie, I C J W Bowler, T E A Peto}

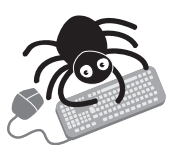

J Clin Pathol 2005;58:352-356. doi: 10.1136/jcp.2004.022293

The supplemental data are available online at http:// www.jclinpath.com/ supplemental

See end of article for authors' affiliations

.....................

Correspondence to: Dr D Wyllie, Nuffield Department of Clinical Laboratory Sciences, John Radcliffe Hospital, Oxford OX3 9DU, UK; david. wyllie@ndcls.ox.ac.uk

Accepted for publication 18 October 2004

\begin{abstract}
Aim: To define the contribution made by $\mathrm{C}$ reactive protein (CRP) measurement to bacteraemia prediction in adults with medical emergencies in the UK.

Methods: This two year cohort study involved 6234 patients admitted as emergency cases to the acute medical or infectious diseases services of the Oxford Radcliffe Hospitals, in whom blood cultures were taken on arrival. The main outcome measures were bacteraemia risk associated with admission CRP concentrations, lymphocyte counts, and neutrophil counts.

Results: The quantitative associations between CRP concentration, admission lymphocyte count, and neutrophil count were defined. Risk of bacteraemia rose continuously as the CRP increased: no "cutoff" value was evident. Models examining combinations of CRP, neutrophil count, and lymphocyte count were developed and validated using a split sample technique. CRP contributed to a model including lymphocyte and neutrophil counts, but its effect was small. CRP alone performed no better than either a model combining lymphopenia and neutrophilia, or than lymphopenia alone.

Conclusions: In patients with acute medical emergencies who are suspected of bacteraemia clinically, CRP concentrations, although associated with bacteraemia, have a limited role in bacteraemia prediction.
\end{abstract}

B acteraemic infection represents the severe end of the spectrum of community acquired infectious disease, and is associated with a high mortality. ${ }^{12}$ Mortality is improved with appropriate treatment, ${ }^{3}$ suggesting that early recognition is a relevant clinical objective.

"We attempted to predict bacteraemia based on $C$ reactive protein, lymphocyte count, and neutrophil count, using a large database of UK emergency admissions"

Experimental data show that the lymphopenia that occurs in sepsis is partly responsible for the outcome of infection. ${ }^{5-7}$ We have recently reported that lymphopenia, and the well known rise in the neutrophil count occurring in sepsis, ${ }^{8}{ }^{9}$ are independently associated with bacteraemia in UK adults with medical emergencies. ${ }^{10}$ In our institution, estimation of the $\mathrm{C}$ reactive protein (CRP) concentration has become very prevalent. Increases in CRP concentration are associated with bacteraemia in patients admitted from the community, ${ }^{11}{ }^{12}$ and in series of hospitalised patients comprising both community acquired and nosocomial infections. ${ }^{13-15}$ We thought that the clinical estimation of bacteraemia risk in the individual patients admitted as an emergency would be aided by an understanding of both the quantitative relation between CRP and bacteraemia, and of how CRP adds to the inferences made from the neutrophil and lymphocyte counts. Because existing data do not address this question, we have attempted to predict bacteraemia based on CRP, lymphocyte count, and neutrophil count, using a large database of UK emergency admissions, derived from a region of low human immunodeficiency virus prevalence.

\section{METHODS}

\section{Study design and setting}

This cohort has been described previously ${ }^{10}$; it comprises consecutive emergency adult medical admissions, aged at least 18 years, admitted from the community to general medical or infectious diseases services of Oxford Radcliffe Hospitals, UK, from l February 1999 to 31 January 2001. Patients admitted to haematology or cardiology wards did not form part of the cohort. Because this represented a retrospective observational study of routinely collected anonymous healthcare records, we did not seek ethical approval for the analysis.

\section{Microbiology, haematology, and CRP estimation}

Blood cultures and full blood counts were processed as described previously. ${ }^{10}$ CRP measurements were performed with a Biostat kit on an Aeroset analyser (Abbott, Maidenhead, Berkshire, UK). This has limits of linearity at 8 and $285 \mathrm{mg} /$ litre; results outside these limits are reported as $<8$ and $>285 \mathrm{mg}$ /litre. Clinical laboratories involved in specimen processing were accredited by the UK Clinical Pathology Accreditation scheme. For the purposes of our study, we considered "significant isolates" as any blood culture yielding an organism other than a coagulase negative staphylococcus or Corynebacterium spp, as described previously. ${ }^{10}$

\section{Data collection and analysis}

Data used in our study were recorded during the patients' admissions on the hospital's information systems, and

Abbreviations: AUC, area under the curve; CRP, $C$ reactive protein; $R O C$, receiver operator characteristic 
Table 1 Blood cultured cases by $C$ reactive protein (CRP) measurement

\begin{tabular}{lllll}
\hline & \multicolumn{1}{l}{ CRP present $(\mathbf{n}=6234)$} & CRP missing $(\mathbf{n}=948)$ & $\mathbf{p}$ Value & t Statistic \\
\hline Age/years & 64.5 & 65.5 & 0.15 & -1.4 \\
Duration of stay/days & 8.89 & 7.95 & 0.012 & 2.5 \\
Haemoglobin $(\mathrm{g} / \mathrm{l})$ & 127 & 126 & 0.13 & 1.5 \\
Lymphocytes $\left(\times 10^{9} / \mathrm{I}\right)$ & 1.27 & 1.26 & 0.89 & 0.1 \\
Neutrophils $\left(\times 10^{9} / \mathrm{I}\right)$ & 9.13 & 9.46 & 0.11 & -1.6 \\
Platelets $\left(\times 10^{9} / \mathrm{I}\right)$ & 279 & 284 & 0.25 & -1.1 \\
Died as inpatient & 0.0993 & 0.1171 & 0.11 & -1.6 \\
\hline
\end{tabular}

abstracted in an anonymous form. Analysis was performed at the end of the study period.

\section{Statistical methods}

SPSS version 11 was used for logistic regression and receiver operating characteristic (ROC) plotting. ROC plots displayed sensitivity versus 1 -specificity, such that areas under the curve (AUC) generated varied from 0.5 to 1.0, with higher values indicating increased discriminatory ability. Confidence intervals on AUCs of ROC plots were calculated using non-parametric assumptions. The odds of significant bacteraemia for a given group of patients were calculated as (cases of significant bacteraemia)/(cases without significant bacteraemia).

To examine the quantitative associations between bacteraemia and neutrophil, lymphocyte, and CRP results, cases were analysed in strata. There was no overlap between the strata. For neutrophils, strata were $0.5 \times 10^{9}$ /litre wide; for lymphocytes, $0.25 \times 10^{9}$ /litre; and for CRP, $10 \mathrm{mg} /$ litre. The odds of bacteraemia were then plotted for each stratum. For CRP, results of $<8$ or $>285 \mathrm{mg} /$ litre were coded as 8 and 285 , respectively.

\section{RESULTS}

\section{Characteristics of the cohort}

There were 21495 cases in the cohort. Of these, 164 cases had missing, or extreme (neutrophils, $>75 \times 10^{9}$ /litre; or lymphocytes $>10 \times 10^{9}$ /litre $)^{10}$ blood count results, leaving 21331 cases for further analysis. Of these, 14149 cases were not blood cultured, whereas $7182(33.6 \%)$ were, a proportion similar to that reported in another European centre. ${ }^{16} \mathrm{~A}$ breakdown of the cases is shown as a flow chart in the supplementary data shown online (fig Sl; http://www. jclinpath.com/supplemental). The patients' ages ranged from 18 to 106 years, and the average inpatient stay was 6.4 days. Of the cases that were blood cultured, 6668 cultures were negative, and 536 yielded significant pathogens, including Escherichia coli (146 cases), other enterobacteriaceae and Pseudomonas spp (73 cases), Staphylococcus aureus (77 cases), Staphylococcus pneumoniae (55 cases), and $\beta$ haemolytic streptococci (33 cases). A more detailed breakdown of this cohort has been published. ${ }^{10}$

The distributions of CRP and white cell counts are shown, by blood culture result, in the supplementary data (table S1; http://www.jclinpath.com/supplemental). In this article, we address the issue of the value of CRP in those patients in whom bacteraemia was suspected clinically, as judged by the taking of a blood culture. For this purpose, 7182 cases were available, and our further analyses concern these. CRP was measured on the day of admission in 6234 cases ( $86.8 \%)$. The patients in whom CRP was measured did not differ from those in whom it was not taken in age, proportion dying in hospital, haemoglobin, and neutrophil, lymphocyte, or platelet counts; however, they did stay one day longer in hospital (table 1). In view of the low proportion (13.2\%) of missing CRP data and their comparable initial parameters, we thought that the 6234 cases were probably an unbiased sample of all blood cultured cases and analysed them further. To construct and validate bacteraemia prediction methods we split the 6234 cases into two, randomly assigning two thirds to a derivation set, and one third to a validation set. The characteristics of these two sets were comparable (table S2; http://www.jclinpath.com/supplemental).

\section{Association between age, bacteraemia, and cell counts}

Using the derivation set of 4185 cases, we analysed the quantitative association between CRP, neutrophil and lymphocyte counts, and bacteraemia. In a preliminary analysis, cases were divided into strata based on their count, and the odds of bacteraemia in each stratum plotted (fig S2; http://www.jclinpath.com/supplemental). Forty patients were neutropenic (neutrophil counts less than $1 \times 10^{9}$ /litre); as expected, their bacteraemia risk was high. For counts above $1 \times 10^{9}$ /litre, the data are compatible with a linear increase in log odds of bacteraemia with rising neutrophil count. This is also the case for CRP concentrations. We coded results as being outside the limit of the assay at the assay limits of 8 and $285 \mathrm{mg}$ /litre; however, deviation of these points from the relation seen within the linearity of the assay is not evident, justifying this approach. For lymphocyte count, the log of the odds of bacteraemia is proportional to the log of the lymphocyte count.

\section{Models of bacteraemia risk}

Using the derivation set, we constructed logistic regression models predicting bacteraemia. Neutropenic patients (neutrophil counts, $<1 \times 10^{9}$ /litre) represent a special case, as described above, and are rare in this cohort; we excluded them from further analysis (fig Sl; http://www.jclinpath. com/supplemental). Because of the linear relation between $\log$ odds of bacteraemia and log lymphocyte count, in our models we used log lymphocyte count, rather than lymphocyte count, as a predictor. By univariate analysis, patient age, neutrophil count, lymphocyte count, CRP concentration, and all interactions between neutrophil count, lymphocyte count, and CRP concentration were significant by forward and backward logistic regression analysis. Table 2 shows the results for backward analysis. We then constructed two logistic regression models: one included all the above terms, and the other omitted terms involving CRP. Age, lymphocyte count, neutrophil count, CRP concentration (when entered), and the lymphocyte-neutrophil interaction were significant by multivariate analysis (table 3 ).

\section{Performance of two models and single variables}

We examined the performance of neutrophil count, lymphocyte count, and CRP concentration, and of the probabilities of bacteraemia calculated using the two models derived from them, in the validation set. ROC curves for each of the five methods in differentiating bacteraemia from non-bacteraemia are presented. Figure 1A shows the curves, fig $1 \mathrm{~B}$ shows 


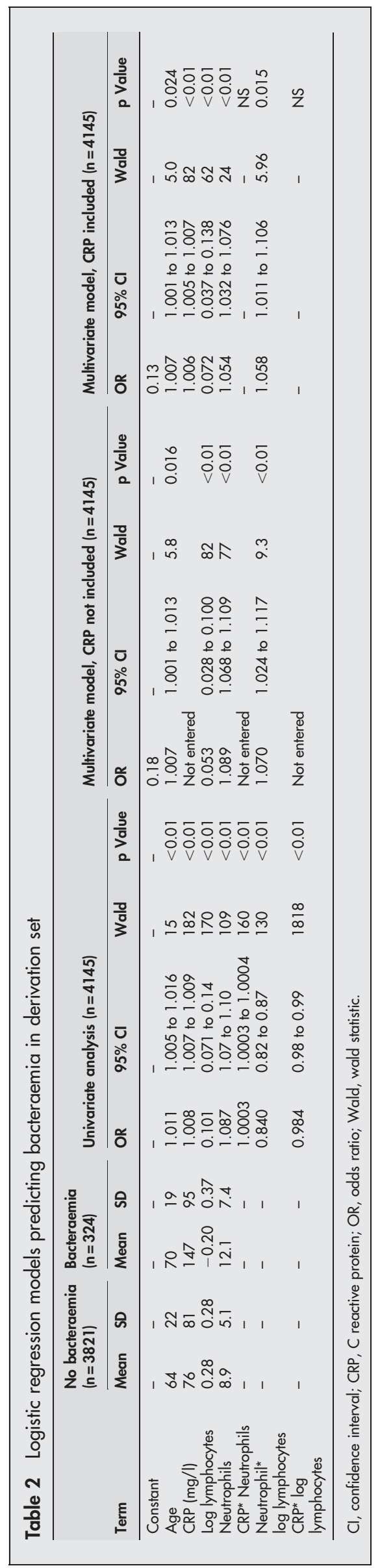

the AUCs with confidence intervals, and fig IC shows the significance of pairwise comparisons of the differences between curve areas. The model formed by CRP, neutrophil count, and lymphocyte count has the highest AUC of 0.78. A model comprising lymphocyte and neutrophil counts has a comparable performance (AUC $=0.75$ ) to the model including all three variables ( $\mathrm{AUC}=0.78$; $\mathrm{p}$ for difference between AUCs, 0.15) and to CRP (AUC $=0.72 ; p$ for difference between AUCs, 0.24).

\section{Likelihood ratios for bacteraemia}

An alternative method of looking at these data, and one that is more useful when faced with an individual patient, is to examine the likelihood ratio for bacteraemia associated with the models and variables studied. The likelihood ratio is the factor relating the pre-test to the post-test odds ${ }^{17}$; we calculated the observed likelihood ratios for a range of parameter values within the derivation set (fig 2). Neutrophil count, lymphocyte count, and both the models derived can generate likelihood ratios of $>10$ for bacteraemia, although the number of cases in which this occurs is small (table 3 ). The peak likelihood ratio achieved by CRP concentrations is less, at 4.0, and occurs with concentrations of $>285 \mathrm{mg} / \mathrm{litre}$.

\section{DISCUSSION}

To our knowledge, this is the first study to assess the quantitative association between bacteraemia, white blood cell components, and CRP, even though alterations in white blood cell distribution and CRP are regarded as classic markers of inflammation. We show that CRP, lymphopenia, and neutrophilia independently predict bacteraemia, and we describe the associations between the values of these parameters and the risk for bacteraemia. Models are derived and validated that further increase the predictive value of these tests. Evidence based medicine theory ${ }^{17}$ suggests that tests with likelihood ratios of 10 or over are those most helpful clinically; likelihood ratios over 10 are only achieved in about $2.7 \%, 2 \%$, and $1 \%$ of cases using our bacteraemia models, neutrophilia, or lymphopenia respectively; they are never achieved using CRP as an infection marker, the peak likelihood ratio achievable with which is 4.0. Thus, although the models we present may be of substantial use to clinicians in some cases, in others their clinical usefulness is uncertain. The information added by CRP to the examination of neutrophil and lymphocyte counts is small.

\section{"In the early detection of the most severe, bacteraemic forms of community acquired infection, the role of $C$ reactive protein may be limited"}

We chose to study bacteraemia as an outcome measure because it represents a dichotomous, objective outcome associated with mortality and with the most severe forms of community acquired sepsis. However, bacteraemia has several limitations as an outcome measure. First, it is not a sensitive measure of adverse outcome, ${ }^{18}$ and the prediction of non-bacteraemic infection may be as important as bacteraemic infection. Our data do not address the issue of whether our model would adequately predict severe non-bacteraemic infections. Neither do they address whether it adds to clinically discernable factors, such as the presence of shock, which are associated with the severity of infection and with outcome. ${ }^{18} 19$

In our hospital, $86 \%$ of blood cultured patients had CRP estimations performed. They were also performed in $70 \%$ of those who were not blood cultured; overall, this suggests that CRP is now regarded as a routine test in this setting, into which considerable resources are being directed. There may 
Table 3 Proportions of cases with high likelihood ratios

\begin{tabular}{|c|c|c|c|c|c|c|}
\hline Analysis of derivation set $(n=2049)$ & $\begin{array}{l}\text { Cutoff value for } \\
L R>5\end{array}$ & $\begin{array}{l}\text { Cutoff value for } \\
L R>10\end{array}$ & $\begin{array}{l}\mathrm{LR}>5 \\
\mathrm{n}=\%\end{array}$ & LR $>5 \%$ & $\begin{array}{l}\mathrm{LR}>10 \\
\mathrm{n}=\%\end{array}$ & LR $<10 \%$ \\
\hline Modelled probability, CRP included & 0.197 & 0.304 & 168 & $8.2 \%$ & 57 & $2.8 \%$ \\
\hline Modelled probability, CRP excluded & 0.181 & 0.250 & 156 & $7.6 \%$ & 53 & $2.6 \%$ \\
\hline Neutrophil count $\left(\times 10^{9} / \mathrm{I}\right)$ & 20.7 & 26.2 & 92 & $4.5 \%$ & 39 & $1.9 \%$ \\
\hline Lymphocyte count $\left(\times 10^{9} / \mathrm{I}\right)$ & 0.263 & 0.149 & 70 & $3.4 \%$ & 23 & $1.1 \%$ \\
\hline CRP (mg/l) & - & - & 0 & $0.0 \%$ & 0 & $0.0 \%$ \\
\hline
\end{tabular}

This table shows the proportion of blood cultured cases in the validation set in which likelihood ratios of 5 and 10 for bacteraemia are predicted by the two models derived here, and by neutrophil count, lymphocyte count, and CRP protein when used as single variables.

$C R P, C$ reactive protein; $L R$, likelihood ratio.

be indications for measuring CRP in acute medical patients, such as monitoring the progression of illness. ${ }^{20-22}$ However, in the early detection of the most severe, bacteraemic forms of community acquired infection, the data presented here suggest that its role is limited. If the detection of severe infectious illness is the aim of using an acute inflammatory marker, and a full blood count is available, one needs to consider whether expending resources on CRP estimation is worthwhile. Other markers may offer better performance. In particular, a substantial literature is emerging on the use of procalcitonin. Studies in emergency departments in Taiwan, ${ }^{23}$ France, ${ }^{24}$ and Turkey ${ }^{25}$ were performed on populations that are probably similar to our blood cultured cohort. Inclusion criteria included having symptoms or signs compatible with infection, ${ }^{23}$ systemic inflammatory respiratory syndrome, ${ }^{25}$ or having a CRP done. ${ }^{24}$ The outcome measures in these studies also varied, but included clinical definitions of infection, ${ }^{23}{ }^{24}$ systemic infection, ${ }^{25}$ and septic shock. ${ }^{23}$ All three studies showed that in the diagnosis of systemic infection ${ }^{24}$ and sepsis, ${ }^{23}{ }^{25}$ procalcitonin performed better than CRP. These studies are compatible with studies showing better performance of procalcitonin than CRP in the prediction of bacteraemia, ${ }^{11}$ and of pneumonia severity, ${ }^{26}$ and with studies from intensive care units showing that procalcitonin is

\section{Take home messages}

- In patients with acute medical emergencies who are suspected of bacteraemia clinically, C reactive protein (CRP) concentrations, although associated with bacteraemia, have a limited role in bacteraemia prediction

- The measurement of CRP concentrations in these patients adds little extra information to neutrophil and lymphocyte counts, and may be a waste of resources

- Other markers, such as procalcitonin, may perform better

more closely correlated with the severity of infection than is CRP. ${ }^{27-29}$

This work emphasises the need for clinical studies that document the performance of existing and novel markers of infection in clearly defined populations, so that severe forms of community acquired infection may be recognised and treated rapidly.

\section{ACKNOWLEDGEMENTS}

We thank members of our department for helpful comments.
A ROC curves

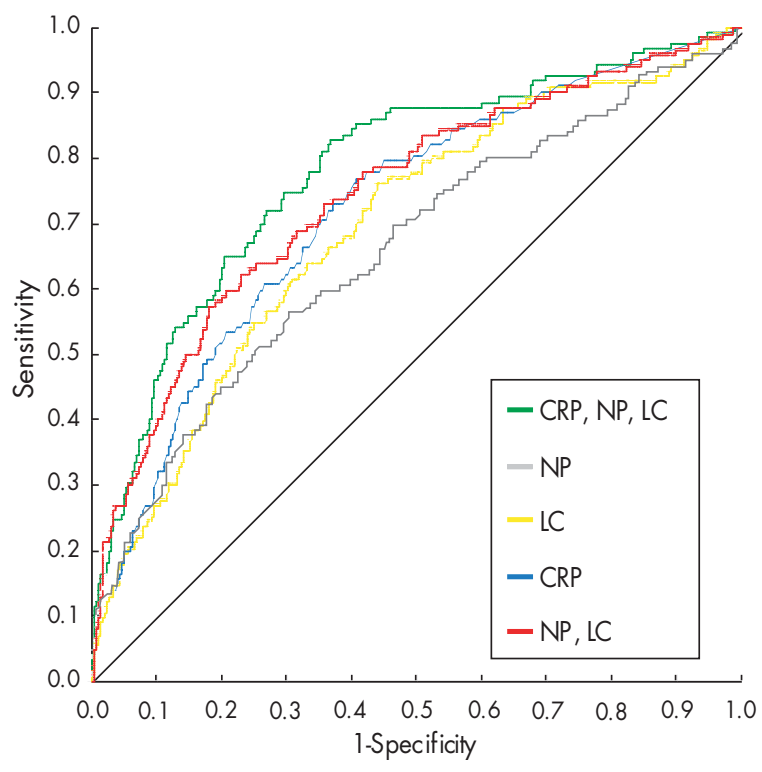

B Areas under ROC curves

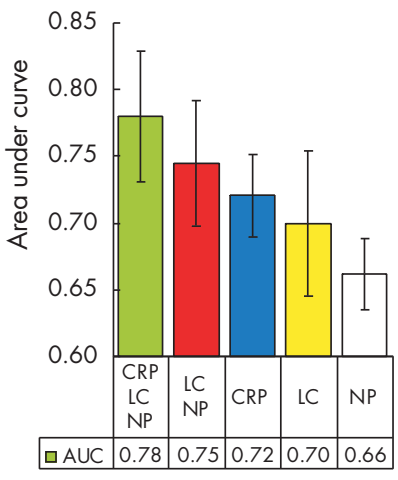

C Differences in ROC areas

\begin{tabular}{c|c|c|c|c}
$p$ & LC, & CRP & LC & NP \\
\hline CRP LC NP & 0.15 & 0.04 & $<0.01$ & $<0.01$ \\
\hline LC NP & - & 0.24 & 0.06 & 0.01 \\
\hline CRP & - & - & 0.23 & 0.05 \\
\hline LC & - & - & - & 0.12
\end{tabular}

Figure 1 This figure illustrates the performance, in the validation of models built using C reactive protein (CRP) concentration, lymphocyte count (LC), and neutrophil count (NP). (A) Receiver operator characteristic (ROC) curves for models built using CRP concentration, lymphocyte count, and neutrophil count (CRP, LC, NP), lymphocyte and neutrophil counts (NP, LC), or single variables. (B) Areas under the ROC curves. (C) The significance of pairwise comparisons between the areas. 
A

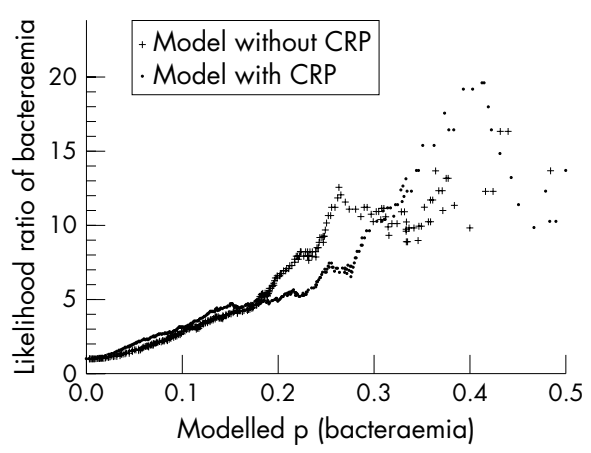

C

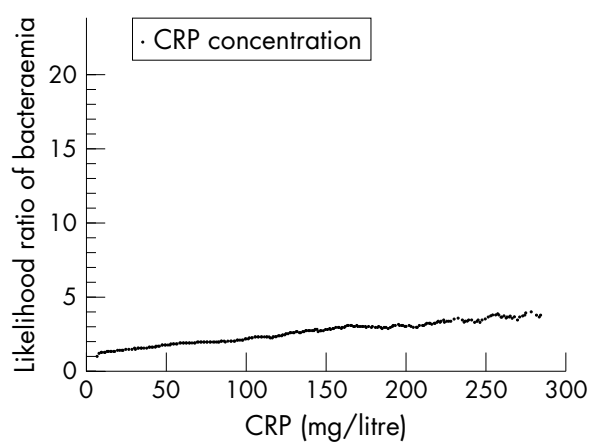

B

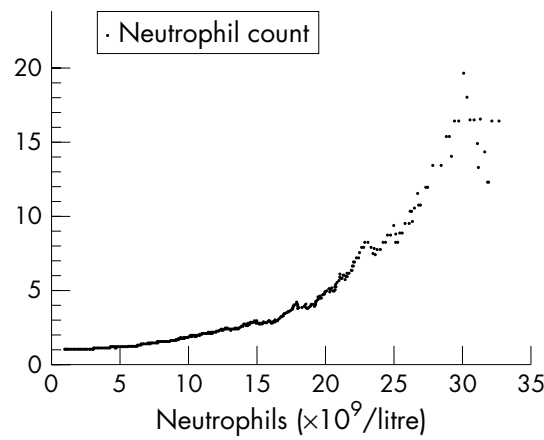

D

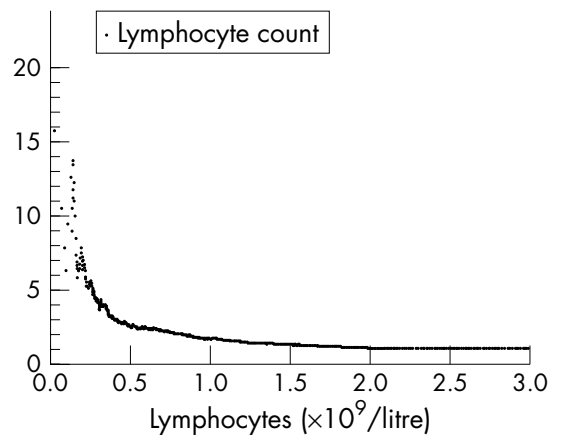

Figure 2 The observed likelihood ratios for bacteraemia associated with the parameters shown in fig 1. (A) The likelihood ratios associated with probabilities of bacteraemia calculated by models without (plus symbols) or with (dots) C reactive protein (CRP). (B-D) Likelihood ratios produced by the range of neutrophil counts, CRP measurements, and lymphocyte counts, respectively.

\section{Authors' affiliations}

TE A Peto, Nuffield Department of Medicine, University of Oxford, John Radcliffe Hospital, Oxford OX3 9DU, UK

D H Wyllie, I C J W Bowler, Nuffield Department of Clinical Laboratory Sciences, University of Oxford, John Radcliffe Hospital

\section{REFERENCES}

1 Mylotte JM, Kahler L, McCann C. Community-acquired bacteremia at a teaching versus a nonteaching hospital: impact of acute severity of illness on 30-day mortality. Am J Infect Control 2001;29:13-19.

2 Bates DW, Pruess KE, Lee TH. How bad are bacteremia and sepsis? Outcomes in a cohort with suspected bacteremia. Arch Intern Med 1995;155:593-8.

3 Hanon FX, Monnet DL, Sorensen TL, et al. Survival of patients with bacteraemia in relation to initial empirical antimicrobial treatment. Scand J Infect Dis 2002;34:520-8.

4 Valles J, Rello J, Ochagavia A, et al. Community-acquired bloodstream infection in critically ill adult patients: impact of shock and inappropriate antibiotic therapy on survival. Chest 2003;123:1615-24.

5 Le Tulzo Y, Pangault C, Gacouin A, et al. Early circulating lymphocyte apoptosis in human septic shock is associated with poor outcome. Shock 2002;18:487-94.

6 Hotchkiss RS, Tinsley KW, Swanson PE, et al. Prevention of lymphocyte cell death in sepsis improves survival in mice. Proc Natl Acad Sci U S A 1999;96:14541-6.

7 Hotchkiss RS, Chang KC, Swanson PE, et al. Caspase inhibitors improve survival in sepsis: a critical role of the lymphocyte. Nat Immunol 2000;1:496-501.

8 Bone RC, Sibbald WJ, Sprung CL. The ACCP-SCCM consensus conference on sepsis and organ failure. Chest 1992;101:1481-3.

9 Shapiro NI, Wolfe RE, Wright S, et al. Who needs a blood culture? A prospectively derived and validated clinical prediction rule. Acad Emerg Med 2003;10:435-6.

10 Wyllie DH, Peto TEA, Bowler ICJ. Relation between lymphopenia and bacteraemia in UK adults with medical emergencies. J Clin Pathol 2004;57:950-5.

11 Chirouze C, Schuhmacher H, Rabaud C, et al. Low serum procalcitonin level accurately predicts the absence of bacteremia in adult patients with acute fever. Clin Infect Dis 2002;35:156-61

12 Rintala EM, Aittoniemi J, Laine S, et al. Early identification of bacteremia by biochemical markers of systemic inflammation. Scand J Clin Lab Invest 2001:61:523-30.

13 McCabe RE, Remington JS. C-reactive protein in patients with bacteremia. J Clin Microbiol 1984;20:317-19.
14 Dryden MS. C-reactive protein concentration in suspected bacteraemia. J Clin Pathol 1986;39:1363-4

15 Byl B, Deviere J, Saint-Hubert F, et al. Evaluation of tumor necrosis factoralpha, interleukin-6 and C-reactive protein plasma levels as predictors of bacteremia in patients presenting signs of sepsis without shock. Clin Microbiol Infect 1997;3:306-16

16 Chassagne $\mathrm{P}$, Perol MB, Doucet J, et al. Is presentation of bacteremia in the elderly the same as in younger patients? Am J Med 1996;100:65-70.

17 Jaeschke R, Guyatt GH, Sackett DL. Users' guides to the medical literature. III. How to use an article about a diagnostic test. B. What are the results and will they help me in caring for my patients? The Evidence-Based Medicine Working Group. JAMA 1994;271:703-7.

18 van Dissel JT, van Langevelde P, Westendorp RG, et al. Anti-inflammatory cytokine profile and mortality in febrile patients. Lancet 1998;351:950-3.

19 van Langevelde P, Joop K, van Loon J, et al. Endotoxin, cytokines, and procalcitonin in febrile patients admitted to the hospital: identification of subjects at high risk of mortality. Clin Infect Dis 2000;31:1343-8.

20 Leaver HA, Yap PL, Rogers P, et al. Peroxides in human leucocytes in acute septic shock: a preliminary study of acute phase changes and mortality. Eur J Clin Invest 1995;25:777-83.

21 Lobo SM, Lobo FR, Bota DP, et al. C-reactive protein levels correlate with mortality and organ failure in critically ill patients. Chest 2003;123:2043-9.

22 Reny JL, Vuagnat A, Ract C, et al. Diagnosis and follow-up of infections in intensive care patients: value of C-reactive protein compared with other clinical and biological variables. Crit Care Med 2002;30:529-35.

23 Chan YL, Tseng CP, Tsay PK, et al. Procalcitonin as a marker of bacterial infection in the emergency department: an observational study. Crit Care 2004;8:R12-20.

24 Hausfater P, Garric S, Ayed SB, et al. Usefulness of procalcitonin as a marker of systemic infection in emergency department patients: a prospective study. Clin Infect Dis 2002;34:895-901.

25 Guven H, Altintop L, Baydin A, et al. Diagnostic value of procalcitonin levels as an early indicator of sepsis. Am J Emerg Med 2002;20:202-6

26 Hedlund J, Hansson LO. Procalcitonin and C-reactive protein levels in community-acquired pneumonia: correlation with etiology and prognosis. Infection 2000;28:68-73.

27 Luzzani A, Polati E, Dorizzi R, et al. Comparison of procalcitonin and $\mathrm{C}$-reactive protein as markers of sepsis. Crit Care Med 2003:31:1737-41

28 Brunkhorst FM, Clark AL, Forycki ZF, et al. Pyrexia, procalcitonin, immune activation and survival in cardiogenic shock: the potential importance of bacterial translocation. Int J Cardiol 1999;72:3-10.

29 Tugrul S, Esen F, Celebi S, et al. Reliability of procalcitonin as a severity marker in critically ill patients with inflammatory response. Anaesth Intensive Care 2002;30:747-54. 